\title{
Deferred-Iteration Aided Low-Complexity Turbo Hybrid ARQ Relying on a Look-up Table
}

\author{
Hong Chen*†, Robert G. Maunder ${ }^{\dagger}$, and Lajos Hanzo ${ }^{\dagger}$ \\ ${ }^{*}$ School of Computer Science and Engineering, \\ University of Electronic Science and Technology of China. \\ ${ }^{\dagger}$ School of ECS, University of Southampton, SO17 1BJ, UK. \\ Tel: +44-23-8059 3125, Fax: +44-23-8059 4508 \\ Email:\{hc07r,rm,lh\}@ecs.soton.ac.uk;
}

\begin{abstract}
Since the introduction of turbo code aided Hybrid Automatic Repeat reQuest (HARQ) schemes, their complexity reduction has drawn research attention. In our previous work, we proposed an Early Stopping (ES) strategy for a turbo HARQ scheme, which results in a beneficial complexity reduction, while maintaining a high throughput. However, this scheme was designed to strike a compromise across the full range of channel conditions. As a further advance, in this paper, we propose a new Deferred Iterations (DI) strategy, which is specifically designed for taking into account the prevalent channel conditions, as characterized by the Extrinsic Information Transfer (EXIT) chart tunnel opening. More specifically, the DI strategy delays the commencement of turbo decoding until an open EXIT chart tunnel appears. Our simulation results demonstrate that the complexity of the proposed DI aided turbo HARQ schemes is reduced by up to $50 \%$, which is achieved without compromising the Packet Loss Ratio (PLR) or throughput.
\end{abstract}

\section{INTRODUCTION}

Turbo codes [1-3] are characterized by an iterative exchange of increasingly reliable soft information between the constituent Bahl, Cocke, Jelinek and Raviv (BCJR) [4] decoders, which are concatenated in parallel and separated by an interleaver. Owing to their near-capacity performance, turbo codes can be successfully combined with Hybrid Automatic Repeat reQuest (HARQ) schemes $[5,6]$, in order to achieve a high throughput. In these turbo HARQ schemes, the transmitter continually transmits turbo-encoded Incremental Redundancy (IR) to the receiver, where BCJR decoding operations may be performed iteratively following the reception of each transmission. Once the decoded message successfully passes the Cyclic Redundancy Check (CRC), the receiver returns an ACKnowledgement $(\mathrm{ACK})$ to the transmitter, in order to cease its transmission of IR.

The early turbo HARQ schemes [6,7] aimed for minimizing the number of IR transmissions required, hence maximizing the throughput. This was achieved by performing a sufficiently high number of BCJR operations following each and every IR transmission in order to ensure that iterative decoding convergence had been achieved. As a result, our previous solution [8] reduced the decoding complexity of the approaches of $[6,7]$ by proposing an Early Stopping (ES) strategy for a turbo HARQ scheme. Like the schemes of $[6$, 7], that of [8] activates iterative decoding following the reception of each transmission. However, the ES strategy of [8] entirely curtails the iterative decoding process, when the rate of iterative Mutual Information (MI) improvement becomes lower than a predetermined threshold. Since this approach eradicates unnecessary decoding iterations, the scheme of [8] exhibited a significantly lower complexity than those of $[6,7]$, without unduly compromising their throughput.

However, in the scheme of [8], the same parameter values were used, regardless of the instantaneous channel conditions, which

${ }^{1}$ Acknowledgements: The research leading to these results has received funding from the European Union's Seventh Framework Programme (FP7/2007-2013) under grant agreement no 214625. The financial support of the China-UK Scholarship Council, of the RC-UK under the IU-ATC as well as the China-UK Science Bridge in $4 \mathrm{G}$ wireless communication initiative is also gratefully acknowledged. imposed a compromise. A quasi-static Rayleigh fading channel model was employed in [8], where the consecutive IR packets experienced uncorrelated fading, while the complex fading envelope within a packet was perfectly static. However, an improved performance may be expected by specifically designing the system parameters for the channel conditions encountered by each transmission packet separately. As described in [9], the channel conditions may be characterized by the open or closed state of the corresponding Extrinsic Information Transfer (EXIT) charts' tunnel, which predicts the evolution of the increasingly reliable extrinsic information that is iteratively exchanged between the components of a turbo decoder. Specifically, for long packets, the emergence of an open tunnel between the EXIT curves implies that a sufficient amount of information has been provided by the channel for a turbo decoder to facilitate a vanishingly low Bit Error Ratio (BER). This is definitely the case, when the decoding trajectory reaches the point of perfect convergence at $(1,1)$ in the EXIT chart. By contrast, errors are likely to be found in the decoded packet, if the tunnel is closed. The complexity of turbo HARQ schemes can be decreased by considering the above-mentioned EXIT tunnel state, since a retransmission will be triggered without performing any decoding operations, if the tunnel is deemed to be closed. The resultant IR transmission may be regarded as a supplement for the insufficient information gleaned from all the previous transmissions. If the IR supplement is adequate, the closed tunnel will become open, allowing the current packet to be successfully recovered. Even if the supplement is 'insufficient', the closed tunnel tends to become more open, since more information is received. Inspired by this rationale, delaying the turbo decoding until an open EXIT tunnel appears has the potential of reducing the decoding complexity of turbo HARQ schemes, since decoding iterations are prevented, until the tunnel remains closed.

In this paper, we propose a new Deferred Iterations (DI) based decoding method. This method requires the determination of the prerecorded minimum MI that must be provided by the $i$ th transmission in order to create an open EXIT tunnel, when combined with all the previous $(i-1)$ pieces of insufficient channel output information. We conceive a Look-Up Table (LUT) to store these minimum MIs. The proposed technique is generically applicable, but in our design example we apply the above-mentioned LUT based DI strategy to a Multiple Component Turbo Code (MCTC) aided HARQ scheme. A MCTC employs the parallel concatenation of more than two BCJR decoders and exhibits an attractive BER performance, even when each component employs the shortest possible generator polynomial of $(2,3)$ [10]. As a result, they are preferred for low-complexity turbo HARQ schemes. Therefore, Section II provides the system model of the LUT based DI strategy aided MCTC HARQ scheme and details the process of determining the EXIT tunnel's open/closed state corresponding to all received transmissions. Section III describes the threshold MI and analyzes the structure of the LUT. Section IV compares the Packet Loss Ratio (PLR), throughput and complexity of a suitably parameterized version of our scheme to those of appropriately chosen benchmarkers. Finally, Section V offers our 
conclusions.

\section{The LUT BASEd DI AIDED MCTC HARQ SCHEME}

\section{A. System Model}

Figure 1 illustrates the simplified schematic of the DI aided MCTC HARQ scheme, where the detailed structure of the MCTC decoder may be found in [8]. The LUT based DI strategy may be employed to enhance the MCTC decoder of Figure 1, without modifying the MCTC encoder. More specifically, each of the transmitter's transmission comprises an IR packet $\mathbf{b}_{i}$, which is the output of a Unity Rate Code's (URC) [11] encoder for the differently interleaved information bits $\mathbf{a}_{i}$. The transmitter continues to generate this IR, until an ACK is returned by the receiver. Here, the URC is a rate1 recursive convolutional encoder having memory- 1 octal generator polynomials of $(2,3)_{o}$.

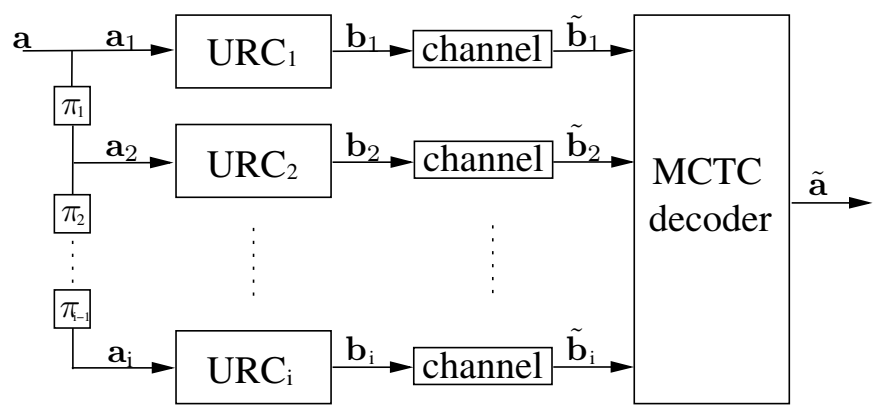

Figure 1. The schematic of the MCTC aided HARQ scheme.

The receiver of the original ES aided MCTC HARQ scheme [8] employs an $i$-component turbo decoder to combine all received Logarithmic Likelihood Ratios (LLRs) $\tilde{\mathbf{b}}_{i}$. However, this iterative decoding process commences immediately after receiving the second transmission $\tilde{\mathbf{b}}_{2}$, since two components are sufficient for constructing an iterative decoder. By contrast, the proposed LUT based DI strategy delays the triggering of iterative decoding, until the EXIT tunnel becomes open. The process of determining whether the tunnel does become open after receiving the $i$ th transmission is represented by the dashed diamond box in Figure 2, which illustrates the flow-chart of the receiver.

In more detail, if the EXIT tunnel is deemed to be sufficiently closed and the retransmission retry limit has not been reached, the receiver idles and waits for the next IR transmission. By contrast, if the tunnel is sufficiently close to becoming open, the $i$-component turbo decoder is activated and iterative decoding proceeds as in the original ES aided MCTC HARQ scheme ${ }^{2}$, starting from the box labeled as 'choose the least recently operated BCJR decoder' in the flow-chart of the decoding process in Figure 3 of [8]. Since no BCJR decoding has been performed at this point, this choice is arbitrary. Furthermore, provided that the EXIT tunnel is open, the iterative decoding process of this MCTC decoder is typically capable of achieving error-free decoding of the current packet. In rare cases, although the EXIT tunnel is marginally open, the MCTC decoder may converge to a legitimate but incorrect decoding decision, which is spotted by the CRC assumed to be perfectly reliable, hence triggering an IR transmission, as detailed in [8]. The packet will be deemed lost, when the number of transmissions reaches a retry limit.

${ }^{2}$ In our LUT based DI aided HARQ scheme, the convergence of the MCTC decoder is defined as reaching the state, when the MI increment of every component BCJR decoder becomes lower than a particular threshold. This is slightly different from the approach of [8], which declares that convergence was reached once any one component decoder satisfies this stopping condition. This modification continues the decoding process for longer and is justified, because the proposed scheme does not commence decoding until there is a good chance that it will become successful.

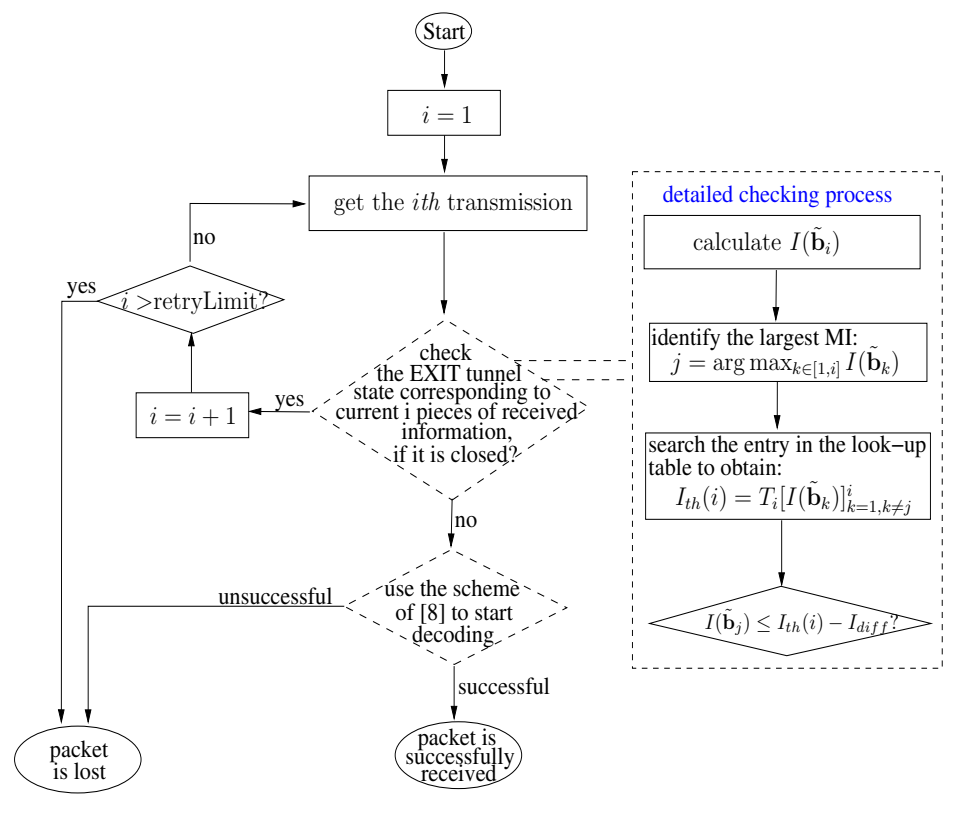

Figure 2. The flow chart of the DI aided MCTC HARQ scheme based on the look-up table.

\section{B. Process of Determining the Open/Closed State of the EXIT Tunnel}

The process of determining the EXIT tunnel's open/closed state is detailed in the dashed rectangle of Figure 2. As seen in Figure 2, when the ith IR transmission is received, the MI $I\left(\tilde{\mathbf{b}}_{i}\right)$ between the received symbols' LLRs $\tilde{\mathbf{b}}_{i}$ and the corresponding bits $\mathbf{b}_{i}$ is calculated using the equation provided immediately beneath Figure 4 in [12], namely from

$$
I(\tilde{\mathbf{x}}) \approx 1-\frac{1}{N} \sum_{j=1}^{N} H_{b}\left(\frac{e^{+\left|\tilde{x}_{j}\right| / 2}}{e^{+\left|\tilde{x}_{j}\right| / 2}+e^{-\left|\tilde{x}_{j}\right| / 2}}\right),
$$

where $\tilde{\mathbf{x}}$ is the $N$-component vector of LLRs, and $H_{b}$ represents the binary entropy function. Next, the index $j$ of the specific HARQ IR transmission attempt having the highest $\mathrm{MI}$ is identified according to $j=\arg \max _{k \in[1, i]} I\left(\tilde{\mathbf{b}}_{k}\right)$. It is this particular received IR sequence $\tilde{\mathbf{b}}_{j}$ that has the highest influence on the success of the entire iterative decoding process and hence it is the corresponding MI $I\left(\tilde{\mathbf{b}}_{j}\right)$ that is used to estimate the EXIT tunnel's open/closed state. This is carried out by checking, whether the most influential MI contribution $I\left(\tilde{\mathbf{b}}_{j}\right)$ is above the threshold $I_{t h}(i)=T_{i}\left[I\left(\tilde{\mathbf{b}}_{k}\right)\right]_{k=1, k \neq j}^{i}$, where the function $T_{i}$ represents the LUT. This $I_{t h}(i)$ is the minimum required MI to create a 'just' open EXIT tunnel, when combined with the other $(i-$ 1) IR contributions. More explicitly, given the knowledge of the most influential MI contribution $I\left(\tilde{\mathbf{b}}_{j}\right)$ so far, the LUT contains statistically relevant information i.e. $I_{t h}(i)$ on whether $I\left(\tilde{\mathbf{b}}_{j}\right)$ may be deemed sufficiently high for ensuring reliable decoding with the additional aid of the other $(i-1)$ IR contributions already received. If so, iterative decoding may be confidently activated rather than delayed, as detailed later in Section III. When the packet length $N$ is sufficiently high, a straightforward comparison between $I\left(\tilde{\mathbf{b}}_{j}\right)$ and $I_{t h}(i)$ may be used to confidently estimate the EXIT tunnel's open/closed state. Specifically, if $I\left(\tilde{\mathbf{b}}_{j}\right) \leq I_{t h}(i)$, the EXIT tunnel is deemed to be closed, otherwise it is deemed to be open.

However, satisfying the condition of $I\left(\tilde{\mathbf{b}}_{j}\right) \leq I_{t h}(i)$ cannot always provide a sufficiently reliable judgement of whether the trajectory can or cannot navigate through the EXIT tunnel to the $(1,1)$ point. This is, because for short packets the trajectory may sometimes navigate through the tunnel that is marginally closed and vice versa [13]. Since our primary objective is to approach the maximum possible throughput, rather than waiting for the EXIT tunnel to open, it is desirable to allow iterative decoding to commence, even if the 
tunnel is marginally closed, especially when the packet length is short. This is achieved by modifying the threshold test according to $I\left(\tilde{\mathbf{b}}_{j}\right) \leq I_{t h}(i)-I_{\text {diff }}$, where $I_{\text {diff }}$ is chosen to be the appropriate MI 'safety margin' for the specific packet length $N$ employed. More particularly, if $I_{\text {diff } f}$ is chosen to be too high, then iterative decoding might commence at too low MI values, when there is no chance for the trajectory to navigate through the tunnel, hence unnecessarily increasing the complexity. By contrast, if $I_{d i f f}$ is chosen to be too low, then iterative decoding will be deferred, even when there is a chance for the trajectory to navigate through the 'just' closed tunnel. This may potentially reduce the throughput. For this reason, we conceived the simulations detailed in Section IV to determine appropriate values for $I_{d i f f}$ for a range of packet lengths.

\section{Construction of the LOOK-UP TABLE}

Given that the MCTC decoder still has a closed EXIT tunnel following the reception of the previous $(i-1)$ LLR sequences having particular MIs, there will be a specific minimum additional MI required for the ith LLR sequence that would allow the EXIT tunnel to open. Therefore, we can define a function $T_{i}$ to describe the relationship between any particular set of $(i-1)$ MIs and the minimum required additional threshold $\mathrm{MI} I_{t h}(i)$ :

$$
I_{t h}(i)=T_{i}\left[I\left(\tilde{\mathbf{b}}_{k}\right)\right]_{k=1, k \neq j}^{i},
$$

where the values of $I\left(\tilde{\mathbf{b}}_{k}\right)$ may be independently spread over the entire legitimate MI range of $[0,1]$. Again, we considered transmissions over quasi-static fading channels, as mentioned in Section I. However, no analytical expression exists for $T_{i}$, since the MCTC decoder employs the iterative BCJR decoding algorithm for exchanging extrinsic information among all the $i$ components. Hence, a LUT is conceived for recording the relationship between the independent and dependent variables of the function $T_{i}$, with a MI granularity of 0.01 .

\section{A. The Threshold MI $I_{t h}(i)$}

The EXIT tunnel's open/closed state is independent of the specific order of receiving its $i$ inputs of IR channel information. For example, assuming that we sequentially receive four transmissions which have the MIs of $I\left(\tilde{\mathbf{b}}_{1}\right)=0.15, I\left(\tilde{\mathbf{b}}_{2}\right)=0.38, I\left(\tilde{\mathbf{b}}_{3}\right)=$ $0.17, I\left(\tilde{\mathbf{b}}_{4}\right)=0.21$, the EXIT tunnel's open/closed state for this 4 component MCTC decoder would remain the same for the sorted reception of $\{0.15,0.17,0.21,0.38\}$. As argued in Section II-B, the highest MI $I\left(\tilde{\mathbf{b}}_{j}\right)=I\left(\tilde{\mathbf{b}}_{2}\right)=0.38$ is compared to the MI threshold of $I_{t h}(4)=T_{4}(0.15,0.17,0.21)$. Given the packet length $N$, the question we ask then is, statistically speaking what is the minimum required next MI contribution for the tunnel to become 'just' open, when we already have the three MI contributions of $\{0.15,0.17,0.21\}$ ? We can answer this question by using the method of drawing EXIT charts for MCTCs, as detailed in our previous work [10]. Specifically for this example, the 4-component MCTC decoder is partitioned into two logical parts. The first one is referred to as the composite decoder, containing three BCJR decoders, which process the received LLRs corresponding to the MIs of $\{0.15,0.17,0.21\}$. The other part is constituted by an individual BCJR decoder, which may scan through the entire input MI range in steps of 0.01 to find a 'just' open EXIT tunnel, when this particular MI is combined with the set $\{0.15,0.17,0.21\}$. More explicitly, the EXIT chart is drawn by exchanging extrinsic information between these two logical parts, where the extrinsic information of the three-component composite decoder is calculated as the sum of extrinsic LLRs gleaned from its constituent BCJR decoders and passed to the individual decoder as a priori information. Quantitatively, when feeding different input MIs to the individual decoder, we found that the EXIT tunnel became 'just' open when $\mathrm{MI}=0.65$. The corresponding EXIT chart is illustrated in Figure 3, where the EXIT tunnel becomes 'just' open between the individual EXIT curve of MI $=0.65$ and the composite
EXIT curve of $\{0.15,0.17,0.21\}$. We note that the unusual shape of the composite EXIT curve of $\{0.15,0.17,0.21\}$ is a consequence of summing the LLRs of the three constituent decoders. Naturally, the tunnel remains closed for all individual EXIT curves having a MI $<0.65$, which is the case for $\mathrm{MI}=0.38$ in Figure 3 .

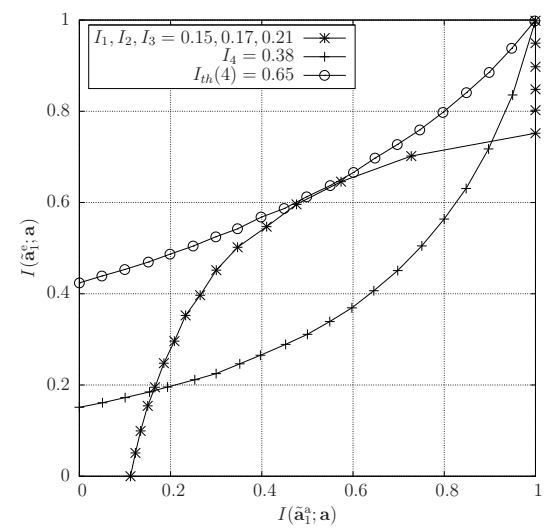

Figure 3. The EXIT chart illustration of $I_{t h}$.

As a result, the EXIT tunnel is deemed to be closed for the fusion of MIs $\{0.15,0.17,0.21,0.38\}$ owing to $0.38<0.65$. Again, the threshold MI of 0.65 used in this example can be pre-recorded in the LUT and found according to the already available MI contributions $\{0.15,0.17 .0 .21\}$ for comparison.

\section{B. The Structure of the Look-up Table}

In order to facilitate an efficient design, the LUT only records the threshold MIs for a limited set of quantized and sorted $(i-1)$ MI values appearing in an ascending order, i.e. satisfying $I\left(\tilde{\mathbf{b}}_{\pi(1)}\right) \leq$ $I\left(\tilde{\mathbf{b}}_{\pi(2)}\right) \leq \ldots \leq I\left(\tilde{\mathbf{b}}_{\pi(i-1)}\right) \leq I_{t h}(i)$, where $\pi$ contains the unique integers of $1, \ldots,(i-1)$ used to appropriately permute the original IR transmission order.

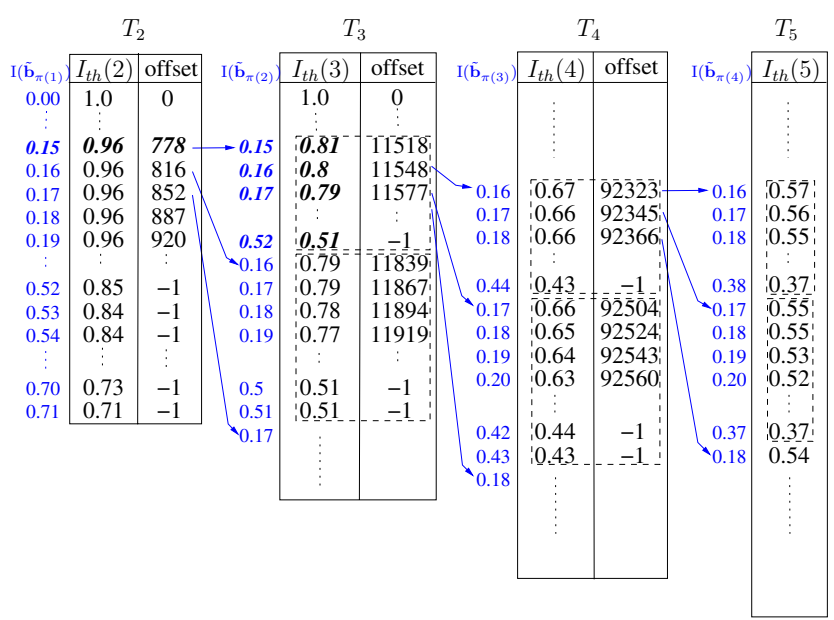

Figure 4. The finally generated LUT for $i=2,3,4$ and 5 , where the input MI granularity is 0.01 .

Figure 4 displays a fraction of the LUT recorded for BPSK transmission over a quasi-static Rayleigh fading channel. As seen in Figure 4, the LUT is composed of several sub-tables, each of which corresponds to Equation 2 for different IR indices $i$. Each sub-table $T_{i}$ - except for the last one - contains two columns. The first one stores the threshold MI $I_{t h}(i)$, while the other indicates the offset of the next sub-table $T_{i+1}$. More explicitly, the first element of each row in the sub-table $T_{i}$ stores $I_{t h}(i)$ valid for a particular set of MIs $\left\{I\left(\tilde{\mathbf{b}}_{\pi(1)}\right), I\left(\tilde{\mathbf{b}}_{\pi(2)}\right), \ldots, I\left(\tilde{\mathbf{b}}_{\pi(i-1)}\right)\right\}$. The second element of each row stores a specific offset of the sub-table $T_{i+1}$, which indicates the starting index of the rows related to the sub-table $T_{i}$ within $T_{i+1}$. Specifically, these rows are displayed in dashed boxes in 
Figure 4, all of which store the threshold MIs $I_{t h}(i+1)$ corresponding to the sets of $\left\{I\left(\tilde{\mathbf{b}}_{\pi(1)}\right), I\left(\tilde{\mathbf{b}}_{\pi(2)}\right), \ldots, I\left(\tilde{\mathbf{b}}_{\pi(i-1)}\right), I\left(\tilde{\mathbf{b}}_{\pi(i)}\right)\right\}$, where $\left.I\left(\tilde{\mathbf{b}}_{\pi(i)}\right)\right\}$ is varied but the previous $(i-1)$ IR MI values remain the same as in conjunction with the equivalent row in $T_{2}$.

Considering $I\left(\tilde{\mathbf{b}}_{\pi(1)}\right)=0.15$ in Figure 4 as an example for further explanations, its corresponding threshold information is $I_{t h}(2)=$ $T_{2}(0.15)=0.96$, implying that the minimum IR MI required for creating an open tunnel is 0.96 , when the received MI of the first transmission is 0.15 . This threshold is stored at the row index of $I\left(\tilde{\mathbf{b}}_{\pi(1)}\right) \cdot 100=15$ in sub-table $T_{2}$, as seen in Figure 4 . Due to the sorting of $I\left(\tilde{\mathbf{b}}_{\pi(1)}\right) \leq I\left(\tilde{\mathbf{b}}_{\pi(2)}\right) \leq I_{t h}(3)$, the initial value of $I\left(\tilde{\mathbf{b}}_{\pi(2)}\right)$ is 0.15 , thence we fix $I\left(\tilde{\mathbf{b}}_{\pi(1)}\right)=0.15$ and increase $I\left(\tilde{\mathbf{b}}_{\pi(2)}\right)$ from 0.15 by 0.01 each step (printed in bold fonts) to explore all possible $I_{t h}(3)$ values corresponding to them. The operations continue until the $I_{t h}(3)$ value required for perfect convergence becomes less than the current $I\left(\tilde{\mathbf{b}}_{\pi(2)}\right)$ value, again, owing to the above-mentioned ordering. The resultant $I_{t h}(3)$ values corresponding to $I\left(\tilde{\mathbf{b}}_{\pi(1)}\right)=0.15$ and the incremental $I\left(\tilde{\mathbf{b}}_{\pi(2)}\right)$ values are recorded in a block of continuous rows, as illustrated in the first dashed rectangle of sub-table $T_{3}$ in Figure 4 . These 38 rows of sub-table $T_{3}$ have the offsets ranging from 778 to 815 , which correspond to the 38 incremental $I\left(\tilde{\mathbf{b}}_{\pi(2)}\right)$ values ranging from 0.15 to 0.52 , as seen in the index area of sub-table $T_{3}$ in Figure 4. Here, the index of this block in sub-table $T_{3}$ starts at 778 , since the rows 0 to 14 in sub-table $T_{2}$ have a total of 778 entries in sub-table $T_{3}$.

There are some special cases to be considered in Figure 4, for example, when the fixed $I\left(\tilde{\mathbf{b}}_{\pi(1)}\right)$ has a larger value, such as $I\left(\tilde{\mathbf{b}}_{\pi(1)}\right)=0.52$. Then, owing to the above-mentioned ordering, the incremental $I\left(\tilde{\mathbf{b}}_{\pi(2)}\right)$ values start from 0.52 . In this situation, the threshold IR MI $I_{t h}(3)=0.15$ corresponding to $\{0.52,0.52\}$ becomes less than the current $I\left(\tilde{\mathbf{b}}_{\pi(2)}\right)=0.52$. This suggests that no entries will be stored in sub-table $T_{3}$ for the fixed $I\left(\tilde{\mathbf{b}}_{\pi(1)}\right)$ value of 0.52 . We tag the corresponding offset as -1 for these cases.

\section{PERFormance Results}

In this section, we evaluate the PLR, throughput and complexity of our previously proposed MCTC HARQ scheme [8] relying on the proposed LUT based DI strategy. This was achieved by simulating the transmission of a statistically relevant number of packets over a Binary Phase-Shift Keying (BPSK)-modulated quasi-static Rayleigh fading channel. We also apply the LUT based DI strategy to Souza's systematic TCTC HARQ [6] and to the LTE system's systematic TCTC HARQ [14]. These three HARQ schemes relying on the ES strategy proposed in [8] are used as our benchmarkers.

Souza's systematic TCTC HARQ transmits the systematic bit sequences $\mathbf{a}$ and the two parity bit sequences $\mathbf{b}_{1}$ and $\mathbf{b}_{2}$. The receiver activates iterative decoding between two parallel connected BCJR decoders after the third IR transmission. From the fourth IR transmission onwards, the repeated frame replica's LLRs are added to those gleaned from the previous transmissions. In contrast to the MCTC HARQ scheme, which may employ a unity-rate accumulator for obtaining the desirable PLR and throughput performances, Souza's HARQ scheme relies on the Recursive Systematic Convolutional (RSC) codes using octally represented memory-3 generator polynomials of $(17,15)_{o}$ for achieving similar results, as seen in [8].

The LTE HARQ scheme adopts RSC codes having different memory-3 polynomials of $(15,13)_{o}$. The LTE standard specifies a particular interleaver, and a so-called 'rate matching' operation for selecting specific transmitted bits rather than transmitting all bits [14]. More explicitly, the standard defines its own interleaver between two parallel concatenated turbo encoders/decoders for a range of specific packet lengths. Furthermore, the systematic bit sequence a and the two parity bit sequences $\mathbf{b}_{1}, \mathbf{b}_{2}$ are interleaved again, according to the standard's so-called sub-block interleavers. The interleaved systematic bits are entered into a circular buffer. The interleaved parity bits fill in the rear part of the circular buffer, where the odd positions are from $\mathbf{b}_{1}$ and the even positions are from $\mathbf{b}_{2}$. Next, $N$ transmitted bits are continuously selected from a specific starting point of this circular buffer. This starting point advances along the circular buffer, based on a standard-specific equation, which is a function of the transmission frame index. As a result, turbo decoding can be activated right after the first frame's transmission, since it contains some of the systematic bits as well as some of the two parity bit sequences. The repeated LLRs are also Chase combined with the corresponding previously received replicas at the receiver.

For each of these HARQ schemes, the transmission retry limit was set to $R=6$ in order to prevent any particular message packet from unduly reserving the network resources, when communicating in hostile environments requiring a high number of retransmissions. For the sake of fair comparison, we increase the maximum number of transmissions defined to be $R=4$ in the LTE HARQ scheme to $R=6$, following the standardized rule of locating the starting point of the circular buffer for each IR transmission. Our results were collected by transmitting source message packets comprising 48 , 480 and 4800 bits over quasi-static Rayleigh fading channels, since these packet lengths can be supported by the LTE HARQ scheme. Additionally, the appropriate $I_{d i f f}$ values were selected for these packet lengths in order to limit the maximum normalized throughput loss imposed by the DI strategy to be as low as 0.003 . Table I shows the preferred $I_{\text {diff }}$ values for the three HARQ schemes considered.

Table I

THE PREFERRED $I_{d i f f}$ VALUES FOR 48,480 AND 4800 BITS PACKET LENGTHS, WHEN THE ALLOWED MAXIMUM THROUGHPUT LOSS IS 0.003.

\begin{tabular}{|c||c||c||c|}
\hline & 48 bits & 480 bits & 4800 bits \\
\hline MCTC HARQ & 0.08 & 0.01 & 0.0 \\
\hline Souza's HARQ & 0.0 & 0.0 & 0.0 \\
\hline LTE HARQ & 0.11 & 0.0 & 0.0 \\
\hline
\end{tabular}

Observe from Table I that for Souza's HARQ scheme, the preferred $I_{\text {diff }}$ values are all zeros regardless of how short the packet length is This is because the determination of the EXIT tunnel's open/closed state only starts after the third transmission, and because there is seldom a 'just' open or 'just' closed EXIT tunnel.

Figure 5 shows the complexity versus SNR performance for the three HARQ schemes both with and without the DI strategy. We employ the same complexity metric as in [8], which was formulated as Complexity $=2^{m} \cdot K$, where $m$ is the number of memory elements employed in the convolutional encoders' generator polynomials and $K$ is the total number of BCJR decoder executions performed during iterative decoding. As shown in Figure 5, the 'MCTC,ES+DI' HARQ scheme offers complexity reductions of approximately $10 \%$, $20 \%$ and $20 \%$ for the packet lengths of 48,480 and 4800 bits respectively, when compared to the 'MCTC,ES' scheme. However, when the LUT based DI is applied to Souza's systematic TCTC HARQ, the complexity reductions become about $35 \%, 32 \%$ and $30 \%$ for the 48, 480 and 4800-bit packet lengths, since Souza's scheme only relied on the ES strategy. Furthermore, the 'LTE,ES+DI' arrangement obtained the highest complexity reductions of up to $50 \%$ for all three packet lengths, since the LTE HARQ scheme activates the turbo decoding right away from the first transmission. The LUTbased DI aided MCTC HARQ scheme shows the lowest complexity among all HARQ schemes.

Let us now define the throughput as the ratio of the number of successfully delivered source message packets to the total number of transmitted packets. The left and right axes of Figure 6 respectively illustrate the PLR and throughput performances, which are similar, regardless of which turbo HARQ scheme is used and whether the DI is employed, for all the three packet lengths considered. There is one exception, where the throughput of the LTE HARQ scheme becomes significantly lower than that of the other two HARQ 


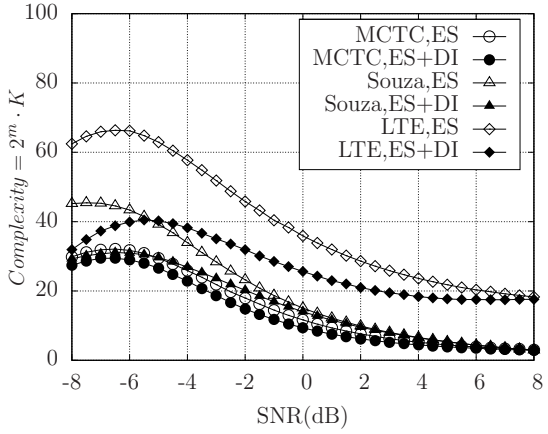

(a) 48bits

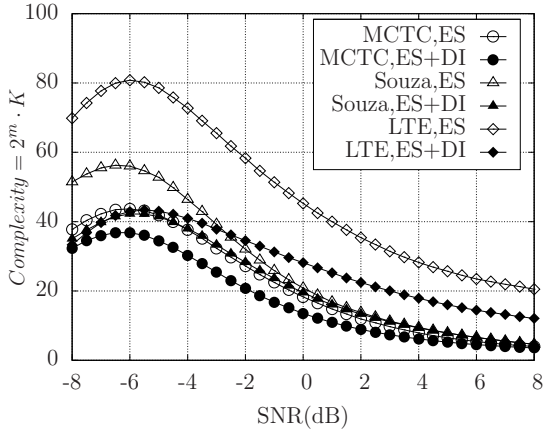

(b) 480 bits

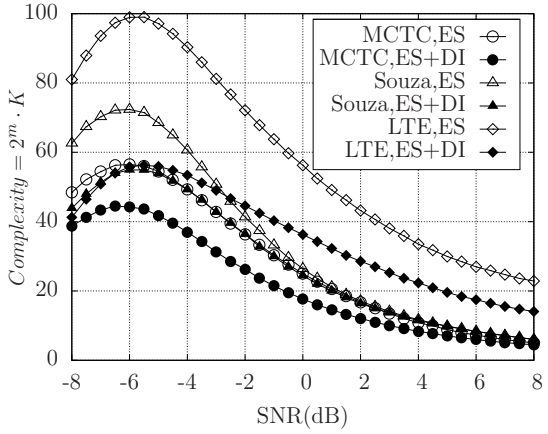

(c) 4800bits

Figure 5. Complexity versus the quasi-static Rayleigh fading channel SNR for message packets of length a) 48 bits, b) 480 bits and c) 4800 bits.

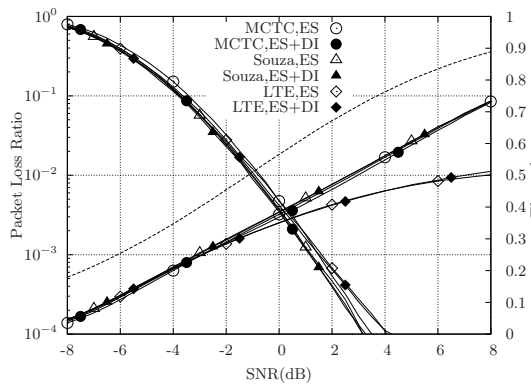

(a) 48bits

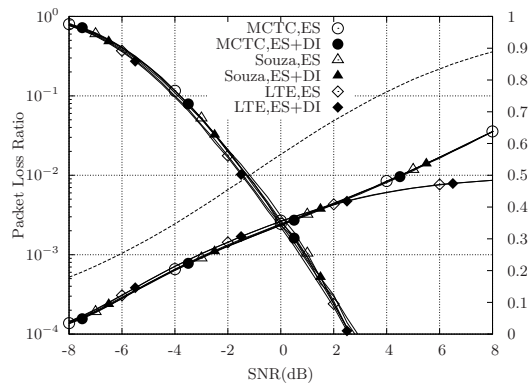

(b) 480 bits

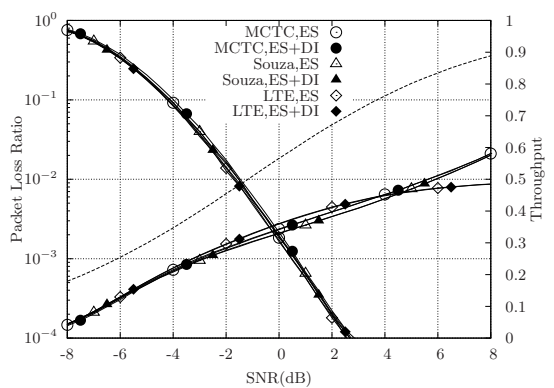

(c) 4800bits

Figure 6. PLR and throughput versus the quasi-static Rayleigh fading channel SNR for message packets of length a) 48 bits, b) 480 bits and c) 4800 bits. The dashed line represents the DCMC capacity.

schemes, namely at high SNRs. This is because many packets may be successfully received after the first transmission attempt in the other two HARQ schemes, while in the LTE HARQ scheme, a minimum of two transmissions are needed for recovering the source packet. Furthermore, the dashed curve seen in Figure 6 reveals the gap between the Discrete-input Continuous-output Memoryless Channel's (DCMC) capacity and the throughput that these three HARQ schemes can achieve.

\section{CONCLUSiOnS}

In this paper, a generically applicable low-complexity DI aided turbo HARQ design was proposed and characterized. Complexity is a critical issue for any communication scheme employing turbo codes, especially for applications like HARQ, which may have to activate iterative decoding multiple times. As demonstrated in [8], the total complexity of the HARQ schemes dispensing with ES strategies may be particularly high. By contrast, the ES strategy aided turbo HARQ scheme of [8] was shown to significantly decrease this complexity. For the sake of decreasing the complexity further, this paper proposed a more sophisticated DI strategy, which exploits the EXIT tunnel's open/closed state for determining, when iterative decoding should commence. At the cost of storing a modest LUT, the proposed scheme has been shown to decrease the complexity by $10 \%$ to $50 \%$ in the context of three recent turbo HARQ benchmarker schemes. This was achieved without imposing a significant degradation upon the throughput or PLR performance. Our future work will consider the application of the proposed DI strategy to other turbo coded schemes.

\section{REFERENCES}

[1] C. Berrou, A. Glavieux, and P. Thitimajshima, "Near shannon limit errorcorrecting coding and decoding: Turbo-codes. 1," in Proceedings of the IEEE International Conference on Communications (ICC), vol. 2, May 1993, pp. $1064-1070$.

[2] L. Hanzo, T. H. Liew, B. L. Yeap, R. Y. S. Tee, and S. X. Ng, Turbo Coding, Turbo Equalisation and Space-Time Coding for Transmission over Fading Channels. John Wiley \& Sons, Ltd, 2011.
[3] L. Hanzo, J. P. Woodard, and P. Robertson, "Turbo decoding and detection for wireless applications," Proceedings of the IEEE, vol. 95, no. 6, pp. 1178-1200, June 2007.

[4] L. Bahl, J. Cocke, F. Jelinek, and J. Raviv, "Optimal decoding of linear codes for minimizing symbol error rate (corresp.)," IEEE Transactions on Information Theory, vol. 20, no. 2, pp. 284 - 287, March 1974.

[5] K. R. Narayanan and G. L. Stuber, "A novel ARQ technique using the turbo coding principle," IEEE Communications Letters, vol. 1, no. 2, pp. 49-51, March 1997.

[6] R. D. Souza, M. E. Pellenz, and T. Rodrigues, "Hybrid ARQ scheme based on recursive convolutional codes and turbo decoding," IEEE Transactions on Communications, vol. 57, no. 2, pp. 315-318, February 2009.

[7] H. Chen, R. G. Maunder, and L. Hanzo, "Multi-level turbo decoding assisted soft combining aided hybrid ARQ," in Proceedings of the IEEE Vehicular Technology Conference Spring (VTC 2010-Spring), May 2010.

[8] — - "Low-complexity multiple-component turbo decoding aided hybrid ARQ," IEEE Transactions on Vehicular Technology, vol. 60, pp. 1571-1577, May 2011.

[9] S. ten Brink, "Convergence behavior of iteratively decoded parallel concatenated codes," IEEE Transactions on Communications, vol. 49, no. 10 , pp. 1727-1737, Oct. 2001.

[10] H. Chen, R. Maunder, and L. Hanzo, "An EXIT-chart aided design procedure for near-capacity N-component parallel concatenated codes," in Proceedings of the IEEE Global Communications Conference, (GLOBECOM), December 2010.

[11] D. Divsalar, S. Dolinar, and F. Pollara, "Serial concatenated trellis coded modulation with rate-1 inner code," in Proceedings of the IEEE Global Telecommunications Conference (GLOBECOM), vol. 2, 27 Nov.-1 Dec. 2000, pp. 777-782.

[12] J. Hagenauer, "The EXIT chart - introduction to extrinsic information transfer in iterative processing," in Proceedings of the 12th European Signal Processing Conference (EUSIPCO), 2004, pp. 1541-1548.

[13] J. Lee and R. Blahut, "Generalized EXIT chart and BER analysis of finite-length turbo codes," in Proceedings of the IEEE Global Telecommunications Conference, (GLOBECOM), vol. 4, 1-5 2003, pp. 2067 2072 vol.4.

[14] "3rd Generation Partnership Project; Technical Specification Group Radio Access Network; Evolved Universal Terrestrial Radio Access (E-UTRA); Multiplexing and channel coding (Release 10)," 3GPP TS 36.212, December, 2010, Downloadable at http://www.3gpp.org/FTP/Specs/html-info/26346.htm. 